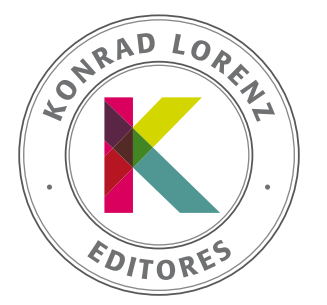

\title{
SUMA PSICOLÓGICA
}

\section{Actitudes hacia el endeudamiento hedónico en estudiantes secundarios chilenos}

\author{
Felipe Quintano Méndez a , Marianela Denegri Coria ${ }^{a, b, *}$
}

\author{
${ }^{a}$ Centro de Excelencia en Psicología Económica y de Consumo, Universidad de la Frontera, Chile \\ ${ }^{b}$ Núcleo Científico Ciencias Sociales, Universidad de la Frontera, Chile
}

Recibido el 13 de octubre de 2020; aceptado el 29 de abril de 2021

\begin{abstract}
PALABRAS CLAVE: Valores materiales, susceptibilidad al efecto de pares, actitudes hacia el dinero, consumo en adolescentes, psicología económica
\end{abstract}

\section{KEYWORDS:}

Material values, influence of peers, attitudes towards money, adolescents consumption, economic psychology

\begin{abstract}
Resumen Introducción: El consumo se ha instaurado como medio de interacción social generando altos niveles de endeudamiento en la población chilena. Los adolescentes han sido especialmente sensibles a esto, producto que a través del consumo acceden a elementos simbólicos asociados a la construcción de la identidad. El objetivo de la presente investigación es determinar la existencia de relaciones entre valores materiales, susceptibilidad al efecto de pares y actitudes hacia el dinero con respecto a las actitudes hacia el endeudamiento hedónico en estudiantes secundarios chilenos. Método: La muestra es de 1297 estudiantes secundarios chilenos, el $46 \%$ es hombre y el $54 \%$ es mujer, correspondientes a las ciudades de La Serena, Coquimbo, Santiago y Temuco. Se aplicaron las escalas de Actitudes hacia el Endeudamiento, Materialismo para Adolescentes, Escala de Susceptibilidad a la Influencia de los Pares y la Escala de Actitudes hacia el Dinero. Los datos fueron analizados a través de una regresión lineal múltiple jerárquica. Resultados: Se evidencian relaciones estadísticamente significativas entre sexo, valores materiales, susceptibilidad al efecto de pares y las actitudes hacia el dinero con respecto al endeudamiento hedónico. Conclusiones: Los resultados obtenidos en este estudio entregan pistas al momento de abordar la educación financiera, donde debe considerarse que la cultura materialista del modelo económico neoliberal impacta en las actitudes, valores y propensión al endeudamiento de los adolescentes y posteriormente adultos.

(c) 2021 Fundación Universitaria Konrad Lorenz. Este es un artículo Open Access bajo la licencia CC BY-NC-ND (http://creativecommons.org/licenses/by-nc-nd/4.0/).
\end{abstract}

\section{Attitudes towards hedonic indebtedness in Chilean high school students}

Abstract Introduction: Consumer spending has established itself as a means of social interaction, generating high levels of indebtedness among the Chilean population. Adolescents have been especially sensitive to this situation, since through consumption they have access to symbolic elements associated with the construction of identity. The objective of this research is to determine the existence of relationships between material values, influence of peers,

\footnotetext{
* Autora para correspondencia.
}

Correo electrónico: marianela.denegri@ufrontera.cl 
and attitudes towards money with regard to attitudes towards hedonic indebtedness in Chilean high school students. Method: Of a sample of 1297 Chilean high school students, $46 \%$ are men and $54 \%$ are women, corresponding to the cities of La Serena, Coquimbo, Santiago and Temuco. The scales of Attitudes towards Indebtedness, Materialism for Adolescents, Scale of Susceptibility to the Influence of Peers and the Scale of Attitudes towards Money were applied. The data were analyzed through a hierarchical multiple linear regression. Results: The results indicate that there is a statistically significant relationship between sex, material values, peer effect and attitudes towards money with respect to hedonic indebtedness. Conclusion: The results obtained in this study provide clues when approaching financial education in adolescents, where it must be considered that the materialistic culture of the neoliberal economic model impacts on the attitudes, values and propensity to indebtedness of adolescents and later adults.

(c) 2021 Fundación Universitaria Konrad Lorenz. This is an open access article under the CC BY-NC-ND license (http://creativecommons.org/licenses/by-nc-nd/4.0/).

Las sociedades actuales se caracterizan por la importancia que se le otorga al consumo como articulador de la vida personal y social, y como una forma de respuesta a la incertidumbre de vivir en las sociedades neoliberales del capitalismo globalizado (Moulián, 1998). Chile fue uno de los países más ortodoxos en el mundo para poner en práctica un conjunto de medidas económicas asociadas al modelo neoliberal, lo que produjo un rediseño de las fronteras entre el mercado y el Estado (Araujo \& Martuccelli, 2013). El consumo se instaló como articulador de la construcción de identidades y elemento central en la definición de estatus, mientras que el crédito se "normalizó" como factor estructurante de las relaciones sociales y de la vida personal al permitir que amplios sectores cumplieran con los mandatos de los ideales neoliberales y satisficieran expectativas más allá de sus recursos objetivos presentes (Ossandón, 2012), de manera que el sujeto participa de una nueva forma de ciudadanía y se transforma así en un ciudadano credit-card (Larraín, 2001).

En este escenario, los adolescentes constituyen un grupo de particular interés para el mercado y sus agresivas estrategias comerciales que influyen en sus patrones de consumo y permiten anticipar comportamientos de endeudamiento futuro (Denegri et al., 2017a; Denegri et al., 2019). Considerando lo expuesto, la presente investigación se propone determinar la existencia de relaciones entre los valores materiales, la susceptibilidad al efecto de pares y las actitudes hacia el dinero con respecto a las actitudes hacia el endeudamiento hedónico en estudiantes secundarios chilenos.

\section{El consumo en la adolescencia}

La adolescencia, en cuanto etapa evolutiva, no surge como una categoría biológica natural como lo es, por ejemplo, la pubertad, sino que es un producto cultural de Occidente vinculado a la necesidad de una mayor preparación por parte de la sociedad burguesa a los individuos jóvenes, para así facilitar su integración a la vida productiva y social. Esto se intensifica en las sociedades neoliberales, donde la construcción de un yo emprendedor se orienta al consumo como expresión y fuente de felicidad, de modo que produce nuevas subjetividades orientadas a las necesidades del mercado (Fernández, 2018; Le Breton, 2014).

Durante este periodo, los jóvenes construyen su identidad independiente y comienzan a tomar conciencia de los modelos de éxito que impone la lógica neoliberal. Desde la infancia ya han sido sometidos a un proceso de socialización económica en el que las actitudes hacia el dinero, el consumo y el materialismo son transmitidas intergeneracionalmente mediante la observación que niños y adolescentes hacen de cómo sus padres hablan del dinero, toman sus decisiones financieras y de endeudamiento (Britt, 2016).

Por otra parte, producto del mismo proceso de individuación y construcción de identidad, los y las adolescentes dependen del juicio externo, especialmente de sus pares, quienes muchas veces influencian sus decisiones de compra (Knežević et al., 2016), ya que en esta etapa son consumidores activos que intervienen en las decisiones de consumo de la familia y, a su vez, manejan un presupuesto propio que administran libremente (Barros et al., 2019; Denegri et al., 2008).

Para Isaksen y Roper (2012), en esta etapa se experimentan altos niveles de inseguridad que llevan a mercantilizar la autoestima con el fin de buscar la estabilidad a través de las oportunidades que les proporciona el consumo y, con ello, instalan pautas de comportamiento como parte de su propia definición identitaria.

Especialmente en las últimas décadas, los jóvenes se han convertido en un foco de la mercadotecnia (Ashraf \& Khan, 2016) tanto para lograr una mayor comprensión de sus comportamientos de compra como a fin de determinar los factores que influyen en su fidelización como compradores, influenciadores y mercado futuro (Aleti et al., 2015; Pillai \& Srivastava, 2015).También los estudios señalan que los adolescentes han sido especialmente afectados por los impactos de la neoliberalización, a causa de que los adultos encargados de su crianza también se han vuelto dependientes del consumo, de manera que educan a las generaciones jóvenes como consumidores y no como ciudadanos (Lapierre \& Rozendaal, 2019).

En síntesis, en la adolescencia la construcción del “yo" a nivel individual y social aparece profundamente ligada a las prácticas de consumo como representaciones de prestigio, poder y acceso a mundos ideales, con lo cual se homogeneizan las aspiraciones entre las distintas clases sociales. Esto instala actitudes positivas hacia el endeudamiento (Castellanos et al., 2020) y cristaliza la influencia de la socialización económica familiar y del entorno de pares de referencia en cuanto a actitudes hacia el dinero y la valorización del materialismo. 


\section{Actitudes hacia el endeudamiento}

Las actitudes hacia el endeudamiento constituyen una posición subjetiva frente al uso del crédito como medio de satisfacción de necesidades personales materiales (Denegri et al., 2011; Godoy et al., 2015). Se han identificado dos perfiles teóricos: el perfil austero y el hedónico (Denegri et al., 2017b; Godoy et al. 2015; Mansilla et al., 2016). Las actitudes hacia el endeudamiento austero señalan una posición cautelosa sobre la administración de los recursos financieros y una postura negativa frente al endeudamiento, mientras una actitud hedonista considera el crédito y el endeudamiento una opción natural para satisfacer de forma inmediata las necesidades materiales.

Se ha observado que los adolescentes muestran comportamiento contradictorio entre prácticas de consumo y el endeudamiento, lo que evidencia comprensión teórica sobre la importancia de la austeridad, pero con prácticas de consumo y uso del dinero poco sistematizadas, sin planificación y con escasa presencia de ahorro, lo cual indica un perfil actitudinal aún no definido, propio de la adolescencia y sus procesos de construcción de la identidad (Denegri et al., 2012b).

Respecto a la postura hedónica frente al endeudamiento, algunos estudios muestran que los individuos endeudados en compras de placer tienden a dar preferencia a estas en el momento de pagar para estar en capacidad de seguir accediendo a estos bienes (Besharat et al., 2015), de forma que se produce una adaptación hedónica que fomenta el gasto excesivo y el endeudamiento. En esta misma línea, otros estudios con adolescentes han mostrado una correlación positiva entre hedonismo y materialismo (Barros et al., 2019). Asimismo, se han encontrado diferencias de género que indican cómo los hombres presentarían actitudes más favorables hacia la deuda (Mansilla et al., 2016; Serido et al., 2014).

\section{Valores materiales}

El materialismo parece haber aumentado a lo largo de las distintas generaciones y ha alcanzado niveles históricamente altos para las actuales generaciones (Twenge \& Kasser, 2013). Una fuente de ello está en el marketing que alienta a los consumidores jóvenes a adoptar valores materialistas que incluso son contrarios a su salud y bienestar (Vandana, 2014).

En la adolescencia, como producto de las presiones de la sociedad de consumo, los objetos materiales cumplen la función de apoyar la construcción de una identidad propia y lograr prestigio frente a los pares, de modo que se vinculan a la autorrealización (Lins et al., 2013). Los valores materiales se definen como la importancia dada a las posesiones en la vida de los individuos basadas en un conjunto de creencias acerca de sus beneficios psicológicos y, especialmente, a la felicidad que proporcionan (Yeniaras, 2016). En la adolescencia se vinculan a las metas (Banerjee \& Dittmar, 2008), de manera que influyen en la capacidad de adaptación social, emocional y conductual (Maras et al., 2015) y se relacionan con el consumo hedónico (János \& Ágnes, 2020). Vinculado a esto las personas con altos valores materiales también valoran el dinero como herramienta que les permite adquirir las posesiones deseadas y muestran, además, actitudes positivas hacia el endeudamiento para lograrlo (Franco de Oliveira, 2020).

\section{Actitudes hacia el dinero}

El dinero como activo material es un tipo de recurso diferente a los bienes porque expresa una potencial promesa de acceso al cumplimiento de las necesidades, en lugar de satisfacerlas directamente (Simmel, 2011). De esta forma, el dinero hace que la gratificación de las necesidades sea posible porque puede ser intercambiado por bienes y servicios, pero también posee funciones simbólicas como un signo de riqueza personal y de éxito (Nelissen \& Meijers, 2011).

En el caso de los adolescentes, tal como lo han planteado diversas investigaciones (Castellanos et al., 2016; Denegri et al., 2012b; Denegri et al., 2010; Denegri et al., 2008), el dinero posee un significado social como medio de integración, pero, a su vez, funciona como diferenciador frente a sus pares.

Las actitudes hacia el dinero se definen como juicios evaluativos hacia el dinero, sus funciones de intercambio, así como significados sociales y culturales, más allá de ser un medio de transacción abstracto (Campos et al., 2015). Los estudios muestran relaciones significativas entre la importancia dada al dinero y el comportamiento hedónico tanto en el consumo como en el endeudamiento (Hoetoro, 2020). Aquellas personas que pagan en efectivo tienden a tener comportamientos más racionales y cuidadosos, los cuales cambian, fundamentalmente, cuando se paga con tarjetas de crédito, en el que prima el gasto hedónico (Tong et al., 2013, Yu et al., 2018).

El materialismo y las actitudes hacia el dinero son entidades diferentes. El materialismo se relaciona con la posesión de bienes materiales que podrían verse como formas "cristalizadas" del potencial inherente al dinero. Por tanto, valorar los bienes no significa necesariamente valorar el dinero de la misma forma (Pilch \& Górnik-Durose, 2017). Al respecto, Tatzel (2002) distingue entre materialistas estrictos en el uso del dinero, preocupados por el costo de los bienes y que cuidan aquellos de mayor valor y aquellos despreocupados con el dinero que compran cosas sin evaluar su costo monetario y las desechan para cambiarlas por otras.

\section{Susceptibilidad al efecto de pares}

La susceptibilidad a la influencia normativa de los pares refleja un deseo de encajar y ser aceptado (Lin \& Chen, 2012), lo cual es una preocupación particularmente importante para los adolescentes. El grupo de referencia de pares también puede actuar como mediador o moderar las decisiones de compra (Fernandes \& Panda, 2019; Gentina, 2014; Lin \& Chen, 2012; Soler et al., 2019), así como los adolescentes van de compra juntos, visitan más lugares y gastan más dinero para responder a la influencia de sus pares.

Al respecto, se aprecia que adolescentes con altos valores materiales se preocupan más por la comparación social, son más susceptibles a la influencia normativa de sus pares, prestan mucha atención a la aprobación o desaprobación de sus compras (Kasser et al., 2007) y comparan más sus posesiones con las de sus amigos (Likitapiwat et al., 2015). 
En esta misma línea, Hoeve et al. (2014), en un extenso metaanálisis, ratifican que los jóvenes con altos niveles de preocupación sobre las opiniones que los demás tienen de ellos, especialmente sus pares, son más propensos a tener deudas en el futuro. En concordancia con lo anterior, los estudios realizados por Castellanos et al. establecen una "correlación positiva con el estilo de compra compulsiva, con la importancia de marca y con la influencia de pares" (2016, p. 14) y entre el materialismo y las actitudes hacia el dinero.

De acuerdo con la revisión precedente, el objetivo general de la presente investigación fue determinar la existencia de relaciones entre los valores materiales, la susceptibilidad al efecto de pares y las actitudes hacia el dinero con respecto a las actitudes hacia el endeudamiento hedónico en estudiantes secundarios chilenos. Considerando los antecedentes, se plantearon las siguientes hipótesis:

$\mathrm{H} 1$ : existe una asociación positiva estadísticamente significativa entre los valores materiales y las actitudes hacia el endeudamiento hedónico.

$\mathrm{H}$ 2: existe una asociación positiva estadísticamente significativa entre la susceptibilidad al efecto de pares y las actitudes hacia el endeudamiento hedónico.

H3: existe una asociación positiva estadísticamente significativa entre las actitudes hacia el dinero y las actitudes hacia el endeudamiento hedónico.

\section{Método}

\section{Selección de la muestra}

Se trabajó con un diseño cuantitativo de tipo correlacional multivariado (Cea, 1996). Se definió como población de estudio a estudiantes de secundaria, hombres y mujeres, que estuvieran asistiendo a establecimientos de dependencia municipalizada, particular subvencionada y particular pagada en Chile de las ciudades de Temuco, Santiago, Coquimbo y La Serena. Esta clasificación responde a la organización administrativa de los establecimientos educacionales chilenos según la entidad sostenedora, las cuales pueden ser municipales con financiamiento de los municipios locales, particular subvencionadas, de sostenedor privado pero con aportes estatales, y particular, en la que el financiamiento es exclusivo de los padres.

La muestra quedó compuesta por 1297 estudiantes, seleccionados según muestreo bietápico por cuotas. La primera etapa fue no probabilística e intencionada con los siguientes criterios de inclusión: (a) matrícula mixta, (b) localización en zona urbana, con matrícula de procedencia urbana al menos del $99 \%$, (c) cursos de primer y segundo año de secundaria, (d) asignación a cursos según criterios históricos y no por rendimiento, (e) índice de vulnerabilidad entre dos desviaciones estándar respecto a la media nacional según su grupo y (f) se procedió a calcular la media de los cinco años anteriores de los resultados de la prueba Simce agrupados según la dependencia. La prueba Simce corresponde al sistema de evaluación que mide el nivel alcanzado correspondiente a los resultados de aprendizaje de acuerdo con el currículum vigente chileno, el cual se aplica a todos los estudiantes tanto a nivel de primaria como de secundaria. Se obtuvieron tres grupos de establecimientos homogéneos. La segunda etapa probabilística simple consideró los tres grupos de establecimientos, de los cuales se seleccionó al azar un tercio de cada tipo de dependencia por ciudad. De los estudiantes incluidos en el estudio, el $46 \%$ es hombre y el $54 \%$ es mujer. Del total de la muestra, el $35.8 \%$ corresponde a la ciudad de La Serena y Coquimbo, el $30.5 \%$ a Santiago y el $33.7 \%$ a Temuco. A su vez, el $30.9 \%$ corresponde a establecimientos con dependencia municipal, el $52.2 \%$ particular subvencionado y el $16.9 \%$ particular pagado.

\section{Instrumentos}

A continuación, se enlistan y describen los instrumentos utilizados.

Escala de Actitudes hacia el endeudamiento. Compuesta por dos subescalas: la primera mide una actitud hacia el endeudamiento hedonista, y la segunda una actitud hacia el endeudamiento austero. Está constituida por 11 ítems en una escala tipo Likert de 4 puntos que va desde Muy de acuerdo hasta Muy en desacuerdo. Estudios anteriores expusieron un alpha de Cronbach de .87 para la dimensión hedonismo y .77 para austeridad (Denegri et al. 2012b).

Escala de Materialismo para Adolescentes. Desarrollada por Goldberg et al. (2003) para ser aplicada a adolescentes hasta los 15 años. La escala es unifactorial y consta de diez ítems medidos en escala de Likert. Su validación a través de un análisis confirmatorio indica un RMSEA de .086.

Escala de Susceptibilidad a la Influencia de los Pares en el Consumo. Desarrollada originalmente por Bearden et al. (1989) para adultos. En este trabajo se emplea la versión reducida para adolescentes adaptada por Zhang (2001) y que utiliza la influencia normativa. Esta escala posee un $\mathrm{CFI}=.93$ y un RMSEA $=.047$.

Escala de Actitudes hacia el Dinero (Luna-Arocas \& Tang, 2004). Originalmente compuesta por 17 ítems en formato Likert, los cuales están organizados en cuatro dimensiones: poder, respeto, felicidad y negativo. En el presente estudio se utilizó la versión para estudiantes chilenos, validada por análisis factorial confirmatorio por Denegri et al. (2012a) y compuesta por dos constructos; felicidad personal ( $\alpha=.82)$ y el constructo poder social $(\alpha=.59)$.

\section{Procedimiento}

El contacto con los establecimientos se dio a través de una carta enviada a los directores de los liceos seleccionados en la que se presentó el objetivo de la investigación y se solicitó autorización para la realización del estudio. Una vez aceptada la participación, se solicitó bases de datos con el listado de estudiantes que se requerían en el propósito de completar las cuotas necesarias en el proceso de muestreo. Con los estudiantes seleccionados se envió un consentimiento informado a sus padres solicitando autorización para sus hijos en el estudio. Luego, se contactó personalmente a los estudiantes con el fin de solicitar la firma de un consentimiento informado que resguardara su información personal. La aplicación del instrumento fue en horario de clases, en la sala de clases, con previo consentimiento de sus respectivos docentes. Todas las aplicaciones tuvieron una duración entre 45 a 60 minutos. 


\section{Procesamiento de la información}

Los datos fueron procesados a través del software SPSS v.23. En primer lugar, se buscaron datos atípicos univariantes y multivariantes por medio de puntajes $Z$ y distancia de Mahalanobis (Myers, 2011). Luego se realizó un análisis exploratorio univariante de los datos utilizando medidas de dispersión, tendencia central y forma. Posterior a esto, se observaron los datos de forma bivariante a través de correlaciones de Pearson con la finalidad de indagar en las relaciones empíricas entre las variables independientes y dependientes. Luego se ejecutó una regresión lineal múltiple jerárquica (Dugard et al., 2010). Comprobados los supuestos de normalidad, independencia de las variables y colinealidad (Hair et al., 1999) se ingresó en el primer bloque el sexo como variable sociodemográfica y, en el segundo bloque, las variables psicológicas vinculadas a la teoría, de modo que se dejó como variable dependiente las actitudes hacia el endeudamiento hedónico. La variable sexo fue codificada en variable dummy, en la cual $0=$ hombre y $1=$ mujer.

\section{Resultados}

\section{Análisis exploratorio de los datos}

Se observa, en primer lugar, que las variables de estudio actitudes hacia el endeudamiento hedónico, valores materiales, susceptibilidad a la influencia de pares y actitudes hacia el dinero (poder y felicidad) presentan una baja dispersión de los datos (tabla 1). Junto con esto existe un bajo nivel de asimetría para todas las variables considerando un rango de 2 a -2 (Gravetter \& Wallnau, 2014). Con respecto a las actitudes hacia el endeudamiento hedónico, los datos se concentran a la izquierda de la curva de distribución normal, caso contrario presentan las variables independientes. En el caso de la curtosis, se presen- tan tantos resultados positivos como negativos. Los datos negativos evidencian una curtosis platicúrtica, es decir, una baja concentración en los valores centrales, mientras los valores positivos denotan una curtosis leptocúrtica, es decir, los datos se concentran en los valores centrales de la variable.

Conjuntamente, en un acercamiento bivariante, se observa que las actitudes hacia el endeudamiento hedónico presentan una baja correlación positiva y estadísticamente significativa con los valores materiales, susceptibilidad a la influencia de pares y actitudes hacia el dinero (poder y felicidad). Por otra parte, los valores materiales poseen una correlación moderada positiva y estadísticamente significativa con la susceptibilidad a la influencia de pares y el dinero en la dimensión felicidad (tabla 2).

\section{Modelo de regresión lineal múltiple jerárquico}

Se evidencia normalidad univariante en los datos al reportar una asimetría entre -2 y 2 (Gravetter \& Wallnau, 2014). Del mismo modo, se establece la independencia de las variables a través del estadístico de Durbin-Watson, el cual se posiciona en 2.102. Finalmente, se aprecia la ausencia de multicolinealidad, reportada a través del estadístico FIV en la tabla 3, el cual establece que este no debe ser superior a 4 . En lo que respecta al modelo, este posee los siguientes estadísticos: ( $g l$ (4.1249), $p<.001, R 2=.07$, $R 2$ ajustado $=.066, F=18.816$ ).

Finalmente, se observan los betas estandarizados, los cuales expresan una asociación estadísticamente significativa entre la variable dependiente y las actitudes hacia el dinero (felicidad), seguido por la susceptibilidad hacia la influencia de los pares, los valores materiales y el sexo vinculado al hombre (tablas 3 y 4 ).

Tabla 1 Estadísticos descriptivos

\begin{tabular}{lccccc}
\hline & $\begin{array}{c}\text { Actitudes hacia } \\
\text { el endeudamiento } \\
\text { hedónico }\end{array}$ & $\begin{array}{c}\text { Valores } \\
\text { materiales }\end{array}$ & $\begin{array}{c}\text { Susceptibilidad } \\
\text { a la influencia de } \\
\text { pares }\end{array}$ & $\begin{array}{c}\text { Actitudes hacia el } \\
\text { dinero (poder) }\end{array}$ & $\begin{array}{c}\text { Actitudes } \\
\text { hacia el dinero } \\
\text { (felicidad) }\end{array}$ \\
\hline Media & 2.225 & 3.011 & 1.929 & 2.123 & 2.000 \\
Mediana & 2.250 & 3.000 & 1.666 & 2.000 & 2.000 \\
Desviación Estándar & .478 & .817 & .862 & .552 & .632 \\
Asimetría & -.323 & .440 & .761 & .126 & .302 \\
Curtosis & -.0107 & -.204 & -.29 & -.248 & -.466 \\
\hline
\end{tabular}

Tabla 2 Correlaciones bivariadas

\begin{tabular}{|c|c|c|c|c|}
\hline & $\begin{array}{c}\text { Valores } \\
\text { materiales }\end{array}$ & $\begin{array}{c}\text { Susceptibilidad a } \\
\text { la influencia de } \\
\text { pares }\end{array}$ & $\begin{array}{l}\text { Actitudes hacia el } \\
\text { dinero (poder) }\end{array}$ & $\begin{array}{l}\text { Actitudes hacia el } \\
\text { dinero (felicidad) }\end{array}$ \\
\hline Endeudamiento hedonismo & $.221^{* *}$ & $.210^{* * *}$ & $.100^{\star *}$ & $.208^{* *}$ \\
\hline Valores materiales & & $.527^{* *}$ & $.281^{* *}$ & $.627^{* *}$ \\
\hline Susceptibilidad hacia la influencia de pares & & & $.253^{* *}$ & $.401^{* *}$ \\
\hline Actitudes hacia el dinero (poder) & & & & $.467^{* *}$ \\
\hline
\end{tabular}

Nota: ${ }^{* *}$. La correlación es significativa en el nivel .01 (bilateral). 
Tabla 3 Coeficientes modelo de regresión lineal múltiple jerárquica

\begin{tabular}{|c|c|c|c|c|c|c|c|}
\hline & \multicolumn{2}{|c|}{$\begin{array}{c}\text { Coeficientes } \\
\text { no estandarizados }\end{array}$} & \multirow{2}{*}{$\begin{array}{c}\text { Coeficientes } \\
\text { estandarizados } \\
\beta\end{array}$} & \multirow[t]{2}{*}{$t$} & \multirow[t]{2}{*}{$p$} & \multicolumn{2}{|c|}{$\begin{array}{c}\text { Estadísticas } \\
\text { de colinealidad }\end{array}$} \\
\hline & $B$ & Error estándar & & & & Tolerancia & VIF \\
\hline $\begin{array}{l}\text { Actitudes hacia el } \\
\text { endeudamiento hedonista }\end{array}$ & 1.828 & .066 & & 27.662 & .000 & & \\
\hline Valores materiales & .058 & .022 & .100 & 2.662 & .009 & .513 & 1.948 \\
\hline $\begin{array}{l}\text { Susceptibilidad hacia la } \\
\text { influencia de pares }\end{array}$ & .063 & .018 & .113 & 3.471 & .001 & .701 & 1.427 \\
\hline $\begin{array}{l}\text { Actitudes hacia el dinero } \\
\text { (poder) }\end{array}$ & -.005 & .027 & -.006 & -.197 & .844 & .774 & 1.291 \\
\hline $\begin{array}{l}\text { Actitudes hacia el dinero } \\
\text { (felicidad) }\end{array}$ & .071 & .029 & .094 & 2.462 & .014 & .507 & 1.971 \\
\hline Sexo & -.054 & .027 & -.056 & -2.032 & .042 & .967 & 1.034 \\
\hline
\end{tabular}

Tabla 4 Simulación de muestreo para coeficientes

\begin{tabular}{|c|c|c|c|c|c|c|}
\hline & & \multirow[t]{2}{*}{ Sesgo } & \multirow{2}{*}{$\begin{array}{l}\text { Error } \\
\text { estándar }\end{array}$} & \multirow[t]{2}{*}{$p$} & \multicolumn{2}{|c|}{$\begin{array}{c}\text { Intervalo de confianza } \\
\text { al } 95 \% \text { de } \mathrm{BCa}\end{array}$} \\
\hline & & & & & Inferior & Superior \\
\hline Actitudes hacia el endeudamiento hedonista & 1.838 & .002 & .068 & .000 & 1.703 & 1.981 \\
\hline Valores materiales & .063 & .000 & .023 & .006 & .018 & .106 \\
\hline Susceptibilidad hacia la influencia de pares & .053 & .000 & .018 & .003 & .018 & .090 \\
\hline Actitudes hacia el dinero (poder) & -.006 & -.001 & .027 & .824 & -.058 & .045 \\
\hline Actitudes hacia el dinero (felicidad) & .068 & .000 & .029 & .019 & .015 & .123 \\
\hline Sexo & -.054 & .000 & .025 & .028 & -.103 & -.005 \\
\hline
\end{tabular}

\section{Discusión}

El objetivo del presente estudio fue determinar la existencia de relaciones entre los valores materiales, la susceptibilidad a la influencia de pares y las actitudes hacia el dinero (poder, felicidad) con respecto a las actitudes hacia el endeudamiento hedónico en estudiantes secundarios chilenos. Los resultados permiten aceptar las hipótesis 1 y 2 . La hipótesis 3 se acepta de manera parcial.

De esta forma, se observa que existe una relación estadísticamente significativa entre los valores materiales y las actitudes hacia el endeudamiento hedonista. Lo anterior se condice con lo establecido por diversos estudios (Badaoui et al. 2018; Denegri et al. 2010; Denegri \& Martínez, 2004) que expresan cómo los adolescentes, al acceder a bienes materiales, acceden a un mundo simbólico en el que el consumo se vincula a la autorrealización en cuanto a función mediadora de la construcción de una identidad individual, y al logro de prestigio frente a los pares, de manera que se relaciona el "amor a las posesiones" y el "amor al dinero" con la felicidad, el éxito y el consumo hedónico en esta etapa (János \& Ágnes, 2020).

Este resultado afirma la relación entre endeudamiento y materialismo (Franco De Oliveira, 2020) y se avanza así a focalizarla en la dimensión de la propensión actitudinal hacia el hedonismo o la búsqueda del placer inmediato en contraposición a la postergación de impulsos.

Se observa relación entre materialismo y susceptibilidad a la influencia ejercida por el grupo de pares, donde aquellos adolescentes con altos valores materialistas también aparecen más preocupados por la comparación social y más susceptibles a la influencia normativa de sus pares, en relación con sus decisiones de compra y consumo (Kasser et al., 2007).

Nuestros resultados evidencian que los estudiantes secundarios chilenos que poseen un mayor nivel de susceptibilidad frente a la influencia de sus pares tienen una mayor tendencia a una actitud hedonista hacia el endeudamiento. Al respecto, según Banerjee y Ditmar (2008), los jóvenes que muestran altos niveles de preocupación por las opiniones que los demás tienen de ellos son más propensos a tener deudas en el futuro, especialmente si su grupo de referencia pertenece a un nivel socioeconómico o de ingresos superior (Gentina 2014; Hoeve et al., 2014). Este complejo sistema de relaciones entre los adolescentes y sus grupos de referencia puede generar incertidumbre y ansiedad al rechazo, de modo que el consumo funciona como regulador emocional en la búsqueda de integración (Isaksen \& Roper, 2012; Jiang et al., 2015; Likitapiwat et al., 2015).

Por su parte, los resultados destacan las actitudes hacia el dinero, en su dimensión felicidad, como estadísticamente significativas y predictoras de actitud hacia el endeudamiento hedonista. Esto concuerda con los hallazgos de investigaciones previas que señalan relaciones entre las actitudes y la importancia dada al dinero y los comportamientos hedónicos a nivel de consumo y endeudamiento. (Hoetoro, 2020; Tong et al., 2013; Yu et al., 2018).

Estos resultados son llamativos y preocupantes si se considera que en la etapa adolescente se instalan de manera progresiva los que serán posteriormente pautas estables de conducta en variados ámbitos, entre estos, el ámbito financiero. 
Esto porque en investigaciones previas se releva que el dinero funciona como diferenciador social, generador de estatus y fuente de satisfacción y felicidad (Castellanos et al., 2020; Denegri et al., 2012a; Denegri et al., 2010; Denegri et al., 2008; Denegri \& Martínez, 2004), y, si esto se vincula a una postura actitudinal hedónica de búsqueda inmediata de satisfacción, puede resultar en un riesgo real de endeudamiento y dificultades financieras en las etapas posteriores en las que tengan acceso a recursos monetarios propios.

Esto configura poco a poco un cuadro poco alentador dado que, como se señaló, se encuentra relación entre los valores materiales y las actitudes hacia el endeudamiento hedonista, lo que, sumado a las valoraciones dadas al dinero como fuente de felicidad y predictor, a su vez, de este tipo de endeudamiento, pareciera configurar la tipología que Tatzel (2002) enunció al describir al grupo de materialistas que utilizan el dinero como un medio inmediato de satisfacción a sus necesidades de compra, sin importar si lo tienen disponible o no, y cómo así usan el crédito para satisfacer esta necesidad hedónica (Tong et al., 2013, Yu et al., 2018).

Nuestros resultados también apoyan lo expuesto por Goldberg et al. (2003) al señalar que en la adolescencia el dinero y su valoración se perciben como medios para lograr la felicidad y adquirir bienes que permiten la aceptación e integración tanto a nivel del yo como de su relación con el grupo de pares. Serán estos bienes materiales propios del mundo simbólico compartido por el grupo de referencia los que le posibilitan ser reconocido como un igual dentro del grupo, reafirmando con ello su identidad.

En lo que respecta a la variable sexo, los resultados expresan una relación estadísticamente significativa entre la variable sexo vinculada al hombre y las actitudes hacia el endeudamiento hedonista, lo cual confirma hallazgos previos en jóvenes universitarios y adultos (Mansilla et al., 2016; Serido et al., 2014). De esta forma, el presente estudio muestra que esta relación entre género y actitudes hacia el endeudamiento se establece tempranamente en la adolescencia y tiende a perpetuarse como parte del comportamiento financiero. En este sentido, se produce una "naturalización" del endeudamiento como una forma aceptada y aceptable de cumplir con los mandatos de la sociedad de consumo, y que en el caso de los hombres se profundiza porque se muestran más materialistas e influenciables por sus pares que las mujeres (Barros et al., 2019). Es hipotetizable que la socialización de género tenga una relación con estos resultados, dado que, a pesar de los cambios culturales, aún se mantienen estereotipos que instalan tempranamente ideales masculinos de éxito vinculado al éxito material y al consumo como vía de realización, poder y felicidad (Le Breton, 2014).

Los resultados obtenidos en este estudio entregan pistas en el momento de abordar la educación financiera en jóvenes, de modo que debe considerarse que la cultura materialista del modelo económico neoliberal impacta en las actitudes, los valores y la propensión al endeudamiento de los adolescentes y, posteriormente, adultos. De esta forma, estos resultados permiten avanzar en relación con los estudios previos al afianzar la relación entre actitudes hacia el dinero en términos de su simbolismo de felicidad como predictor de un tipo específico de endeudamiento que es el hedónico, y cómo esto, además, se asienta en valores materialistas y en la susceptibilidad a la influencia de los pares con una probable relación con la socialización de género, especialmente con los hombres.

Todos estos aspectos deberán ser profundizados en investigaciones posteriores orientadas a modelizar las relaciones entre materialismo, actitudes hacia el dinero, propensión al endeudamiento hedonista y el papel mediador o moderador que podrían tener el género y la susceptibilidad a la influencia de pares.

\section{Reconocimientos}

Esta investigación fue financiada por medio del DIUFRO DI19-0088.

\section{Referencias}

Aleti, T., Brennan, L., \& Parker, L. (2015). Consumer socialisation agency within three-generational Vietnamese families. Young Consumers, 16(2), 172-188. https://doi.org/10.1108/ yc-08-2014-00471

Araujo, K., \& Martuccelli, D. (2013). Individu et néolibéralisme: Réflexions à partir de l'expérience chilienne. Problèmes d'Amérique Latine, 88, 125-143. https://doi.org/10.3917/pal.088.0123

Ashraf, M., \& Khan, K. M. (2016). Adolescents' role in family decision-making for services in India. Young Consumers, 17(4), 388-403. https://doi.org/10.1108/yc-06-2016-00608

Badaoui, K., Lebrun, A., Su, C., \& Bouchet, P. (2018). The influence of personal and social identity on the clothing consumption of adolescents. Canadian Journal of Administrative Sciences, 35(1), 65-78. https://doi.org/10.1002/CJAS.1397

Banerjee, R., \& Dittmar, H. (2008). Individual differences in children's materialism: The role of peer relations. Personality and Social Psychology Bulletin, 34(1), 17-31. https://doi.org/ 10.1177/0146167207309196

Barros, S., Denegri, M., \& Salazar, P. (2019). Consumo, actitudes hacia el endeudamiento, materialismo e influencia de pares en adolescentes rurales del sur de Chile. Interdisciplinaria, 36(1), 203-219.

Bearden, W., Netemeyer, R., \& Teel, J. (1989). Measurement of consumer susceptibility to interpersonal influence. Journal of Consumer Research, 15(4), 473-481. https://doi.org/10.1086/ 209186

Besharat, A., Varki, S., \& Craig, A. W. (2015). Keeping consumers in the red: Hedonic debt prioritization within multiple debt accounts. Journal of Consumer Psychology, 25(2), 311-316. https://doi.org/10.1016/j.jcps.2014.08.005

Britt, S. L. (2016). The intergenerational transference of money attitudes and behaviors. Journal of Consumer Affairs, 50(3), 539-556. https://doi.org/10.1111/joca.12113

Campos, D., Peñaloza, V., Pinto, F., Denegri, M., \& Orellana, L. (2015). Attitudes towards money and motivational orientation to work in Brazilian young workers. Contaduría y Administración, 60(1), 11-30. https://doi.org/10.1016/S0186-1042(15)72145-5

Castellanos, L., Denegri, M., \& Salazar, P. (2020). Adolescentes chilenos: compra, valores materiales y satisfacción con la vida. Redie, 22, 1-11. https://doi.org/10.24320/redie.2020.22. e04.2155

Castellanos, L., Sepúlveda, J., \& Denegri, M. (2016). Análisis teórico de la relación entre estilos de compra, valores materiales y satisfacción con la vida en la adolescencia. Revista de Psicología y Ciencias del Comportamiento de la U.A.C.J.S., 7(1), 1-22. 
Cea, A. (1996). Metodología cuantitativa. Estrategias y técnicas de investigación social. Editorial Síntesis.

Denegri, M., Alí, Í., Novoa, M., Rodríguez, C., Del Valle, C., González, Y., Etchebarne, M. S., Miranda, H., \& Sepúlveda, J. (2012a). Relaciones entre las escalas de actitudes hacia el dinero y la compra: un estudio en Estudiantes de Pedagogía de Chile. Revista Interamericana de Psicología, 46(2), 229-238.

Denegri, M., Barros, S., Cárdenas, V., Sepúlveda, J., \& Vivallo, O. (2017a). Consumo y endeudamiento en adolescentes escolarizados de la Región de La Araucanía, Chile. Estudios Pedagógicos, 53(1), 61-74. https://doi.org/10.4067/ S0718-07052017000100004

Denegri, M., Cabezas, D., Del Valle, C., González, Y., \& Sepúlveda, J. (2012b). Escala de actitudes hacia el endeudamiento: validez factorial y perfiles actitudinales en estudiantes universitarios chilenos. Universitas Psychologica, 497-509.

Denegri, M., Cabezas, D., Páez, A., Sanhueza, O., Vargas, M., \& Sepúlveda, J. (2010). Actitudes hacia el endeudamiento en adolescentes de educación municipal y particular subvencionada de la ciudad de Temuco. Educación y Humanidades, 2(1), 46-63.

Denegri, M., Caro, C., Cerda, M., Eschmann, K., Martínez, M., \& Sepúlveda, J. (2017b). Relación entre actitudes hacia el endeudamiento y discrepancia del yo en estudiantes de pedagogía chilenos. Revista Electrónica "Actualidades Investigativas en Educación”, 17(3), 1-28. https://doi.org/10.15517/AIE. V17I3.29248

Denegri, M., Concha, A., \& Sepúlveda, J. (2019). Adaptation and Validation of the Economic and Financial Literacy Test for Chilean secondary students. Revista Latinoamericana de Psicología, 51(2), 113-122. https://doi.org/10.14349/rlp.2019.v51.n2.6

Denegri, M., Lara, M., Córdova, G., \& Del Valle, C. (2008). Práctica de ahorro y uso del dinero en preadolescentes (Tweers) chilenos. Universum, 1(23), 24-38.

Denegri, M., \& Martínez, G. (2004). ¿Ciudadanos o consumidores? Aportes constructivistas a la educación para el consumo. PAlDEA. Revista de Educación, 37, 101-116.

Denegri, M., Sepúlveda, J., \& Godoy, M. (2011). Actitudes hacia la compra y el consumo de estudiantes de pedagogía y profesores en ejercicio en Chile. Psicología desde el Caribe, 28, 1-23.

Dugard, P., Todman, J., \& Staines, H. (2010). Approaching multivariante analysis: A practical introducción. Routledge.

Fernandes, S., \& Panda, R. (2019). Influence of social reference groups on consumer buying behavior: A review. Journal of Management Research, 19(2), 131-142.

Fernández, D. (2018). Critical discourse analysis of subjective well-being and positive psychology in adolescence. Límite, 13(43), 39-51. https://doi.org/10.4067/S0718-50652018000300039

Franco de Oliveira, S. (2020). Influence of behavioral factors on the propensity for indebtedness of university students. Brazilian Journal of Management. Revista de Administração Da UFSM, 13(4), 829-849. https://doi.org/10.5902/1983465935196

Gentina, E. (2014). Understanding the effects of adolecent girls'social position within peer groups on exchange practices. Journal of Consumer Behaviour, 13, 73-80. https://doi.org/10.1002/ cb. 1460

Godoy, M. P., Araneda, L., Díaz, M., Villagrán, P., \& Valenzuela, S. (2015). Satisfacción con la vida, actitudes hacia la compra y actitudes hacia el endeudamiento. Revista de Investigación en Psicología, 18(1), 29-46. https://doi.org/10.15381/rinvp. v18i1.11771

Goldberg, M., Gorn, G., Peracchio, L., \& Bamossy, G. (2003). Understanding materialism among youth, Journal of Consumer Psychology, 13(3), 278-288. https://doi.org/10.1207/ S15327663JCP1303_09
Gravetter, F., \& Wallnau, L. (2014). Essentials of statistics for the behavioral sciences. Wadsworth.

Hair, J., Anderson, R., Tatham, R., \& Black, W. (1999). Análisis multivariante. Prentice Hall.

Hoetoro, A. (2020). The relationship between love of money, Islamic religiosity and life satisfaction: A Muslim's perspective. Iqtishadia, 13(1), 37-52. https://doi.org/10.21043/iqtishadia. v13i1.7333

Hoeve, M., Stams, G., Zouwen van der, M., Vergeer, M., Jurrius, K., \& Asscher, J. (2014). A systematic review of financial debt in adolescents and young adults: Prevalence, correlates and associations with crime. PLOS ONE, 9(8), 1-16. https://doi.org/ 10.1371/journal.pone.0104909

Isaksen, K., \& Roper, S. (2012). The commodification of self-esteem: Branding and British teenagers. Psychology \& Marketing, 3(29), 117-135. https://doi.org/10.1002/mar.20509

János, D., \& Ágnes, H. (2020). A materializmus, boldogság, siker és élményfogyasztás összefüggéseinek vizsgálata tinédzserek körében. Vezetéstudomány-Budapest Management Review, 51(4), 63-73. https://doi.org/10.14267/VEZTUD.2020.04.06

Jiang, J., Zhang, Y., Ke, Y., Hank, S., \& Qju, H. (2015). Can't buy me frienship? Peer rejection and adolescent materialism: Implicit self-esteem as a mediator. Journal of Experimental Social Psychology, 58, 48-55. https://doi.org/10.1016/j.jesp.2015.01.001

Kasser, T., Cohn, S., Kanner, A., \& Ryan, R. (2007). Some costs of American corporate capitalism: A psychological exploration of value and goal conflicts. Psychological Inquiry, 18(1), 1-22.

Knežević, B., Delić, M., \& Ptić, K. (2016). Clothing buying motives and store selection criteria. The case of croatian adolescents. Posebno Izdanje, 105-116.

Lapierre, M. A., \& Rozendaal, E. (2019). A cross-national study examining the role of executive function and emotion regulation in the relationship between children's television exposure and consumer behavior. Journal of Youth \& Adolescence, 48(10), 1980-2004. https://doi.org/10.1007/s10964-019-01119-7

Larraín, J. (2001). Identidad chilena. LOM.

Le Breton, D. (2014). Una breve historia de la adolescencia. Nueva Visión Argentina.

Likitapiwat, T., Sereetrakul, W., \& Wichadee, S. (2015). Examining materialistic values of university students in Thailand. International Journal of Psychological Research, 8(1), 109-118. https://doi.org/10.21500/20112084.650

Lin, Y., \& Chen, C. (2012). Adolescents' impulse buying: Susceptibility to interpersonal influence and fear of negative evaluation. Social Behaviour and Personality, 40(3), 353-358. https://doi. org/10.2224/sbp.2012.40.3.353

Lins, S., Bottequin, E., Dóka, Á., Golasa, A., Hylander, F., Merchán, A., Odabašić, A., \& Pavlović, S., (2013). To think, to feel, to have: The effects of need for cognition, hedonism and materialism on impulse buying tendencies in adolescents. Journal of European Psychology Students, 4(2), 25-32. https://doi. org $/ 10.5334 /$ jeps.bh

Luna-Arocas, R., \& Tang, T. (2004). The love of money, satisfaction, and the protestant work ethic: Money profiles among university professors in the U. S. A. and Spain. Journal of Business Ethics, 50, 329-354. https://doi.org/10.1023/B:BUSI.0000025081.51622.2F

Maras, P., Moon, A., Gupta, T., \& Gridley, N. (2015). The role of materialism on social, emotional and behavioural difficulties for British adolescents. Emotional \& Behavioural Difficulties, 20(4), 362-380. https://doi.org/10.1080/13632752.2014.9 89055

Mansilla, L., Denegri, M., \& Álvarez, B. (2016). Relación entre actitudes hacia el endeudamiento y locus de control del consumidor en estudiantes universitarios. Suma Psicológica, 23(1), 1-9. https://doi.org/10.1016/j.sumpsi.2015.11.002 
Moulián, T. (1998). El consumo me consume. Santiago: LOM.

Myers, T. (2011). Goodbye, listwise deletion: Presenting hot deck imputation as an easy and effective tool for handling missing data. Communication Methods and Measures, 5, 297-310. https://doi.org/10.1080/19312458.2011.624490

Nelissen, R. M., \& Meijers, M. H. (2011). Social benefits of luxury brands as costly signals of wealth and status. Evolution and Human Behavior, 32, 343-355. https://doi.org/10.1016/j. evolhumbehav.2010.12.002

Ossandón, J. (2012). Destapando la caja negra. Sociología de los créditos de consumo en Chile. Santiago de Chile: ICSO; Universidad Diego Portales.

Pilch, I., \& Górnik-Durose, M. (2017). Grandiose and vulnerable narcissism, materialism, money attitudes, and consumption preferences. Journal of Psychology, 151(2), 185-206. https:// doi.org/10.1080/00223980.2016.1252707

Pillai, S., \& Srivastava, A. (2015). Consumer decision-making styles of Indian adolescents. Contemporary Management Research; Sansia, 11(4), 385-408. https://doi.org/10.7903/cmr.14181

Serido, J., Shim, S., Xiao, J. Tang, C., \& Card, N. (2014). Financial adaptation among college students: Helping students cope with financial strain. Journal of College Student Development, 55(3), 310-316. https://doi.org/10.1353/CSD.2014.0032

Simmel, G. (2011). The philosophy of money. Routledge.

Soler, F., Bustos, J., Palacios, J., Zeelemberg, M., \& Díaz, R. (2019). Development and validation of the Inventory of Emotional and Reasoned Purchases Decision-Making Styles (PDMI). Suma Psicológica, 26(2), 75-85. https://doi.org/10.14349/sumapsi.2019. v26.n2.3
Tatzel, M. (2002). "Money worlds" and well-being: An integration of money dispositions, materialism and price-related behavior. Journal of Economic Psychology, 23, 103-126. https://doi.org/ 10.1016/S0167-4870(01)00069-1

Tong, L., Zheng, Y., \& Zhao, P. (2013). Is money really the root of all evil? The impact of priming money on consumer choice. Springer Science+Business Media.

Twenge, J., \& Kasser, T. (2013). Generational changes in materialism and work centrality, 1976-2007: Associations with temporal changes in societal insecurity and materialistic role modeling. Personality and Social Psychology Bulletin, 39(7), 883-897. https://doi.org/10.1177/0146167213484586

Vandana, U. (2014). A review on the role of media in increasing materialism among children. Procedia. Social and Behavioral Sciences, 133, 456-464. https://doi.org/10.1016/j. sbspro.2014.04.212

Yeniaras, V. (2016). Unpacking the relationship between materialism, status consumption and attitude towards debt: The role of Islamic religiosity. Journal of Islamic Marketing, 7(2), 232247. https://doi.org/10.1108/JIMA-03-2015-0021

Yu, H., Zhang, R., \& Liu., B. (2018). Analysis on consumer's purchase and shopping well-being in online shopping carnivals with two motivational dimensions. Sustainability, 10(12), 1-18. https://doi.org/10.3390/SU10124603

Zhang M. (2001). Differences in susceptibility to interpersonal influence-Chinese s-generation adolescents and older adults (tesis de maestría). ON, Guelph, Canadá. 